\title{
ALTERACIONES FÍSICO-QUÍMICAS EN EL RÍO IREGUA (LA RIOJA) DEBIDAS A LA CONSTRUCCIÓN DEL EMBALSE DE PAJARES
}

\author{
J. Martínez Abaigar, E. Núñez Olivera, R. Tomás Las Heras, N. Beaucourt \& A. García Álvaro \\ Área de Biología Vegetal, Universidad de La Rioja, Avenida dc la Paz 105, 26004 Logroño, España
}

Key words: physico-chemical characteristics, reservoirs, rcgulated rivers. Iregua rivcr. La Rioja

Palabras clave: características físico-químicas, einbalses, ríos regulados, río Iregua, La Rioja.

\begin{abstract}
PHYSICOCHEMICAL CHANGES IN THE IREGUA RIVER (LA RIOJA) AS A CONSEQUENCE OF THE BUILDING OF PAJARES RESERVOIR

The cnvironmental impact that the building of the Pajares reservoir, started in 1986, has caused on the physical and chemical charactcristics of the Iregua river (La Rioja, Spain) has been analyzed. Physicochcinical data of the sampling stations of Islallana (580 in on the Iregua river) and Torrcmontalbo (429 $\mathrm{m}$ on ihc Najerilla rivcr, control station) during the period 1981-1995 have bccn utilizcd.

Changes reportcd for most of the variables are explained by the influence of geographical, clirnatic, topographical or seasonal factors. Howcver, the outstanding iricreasc in suspended material in Islallana frorn 1991 onwards is probably due to the building of the reservoil
\end{abstract}

\section{INTRODUCCIÓN}

Los recursos hídricos en España, especialinente en su área biogeográfica mediterránea. son escasos y altamente variables en el espacio y el tiernpo. Esta es una de las razones principales de la construcción de embalses, que comenzó en tiempos históricos y que ha alcanzado su ináxirno desarrollo a lo largo del presente siglo, de rnancra paralela al progreso económico y a las mejoras tecnológicas. Actualmente, España cuenta con unos 1.000 cmbalses, que acumulan una capacidad total cercana a los $45.000 \mathrm{Hm}$ (RIERA et al., 1992). Sus usos incluyen tanto el suministro de agua y el riego agrícola, como el control del caudal y la producción de electricidad.

La investigación limnológica sistemática de los embalses españoles comenzo a finales de la década de los 60 , y registró un extraordinario avance con el estudio regional de MARGALEF et al. (1976), actualizado y revisado posteriorrneritc por RIERA et al. (1992). Estos trabajos analizan las características físico-químicas y morfornétricas de ios embalses, así como la abundancia y distribución de los organismos planctónicos (fitoplancton, roíleros y crustáccos). Sin embargo, ningún embalse riojano fue incluido cn dichos estudios, por lo que se carecc de información ecológica de primera mano sobre los cinbalses regionales.

Como consecuencia del gran número de embalses existentes en España. todos los grandes ríos y la inayoía de los cursos fluviales dc tarnaño medio están regulados (GARCÍA DE JALÓN et al., 1992). En España se ha prestado rnuy poca atención a los cfectos de la regulación fluvial sobre la limnología bísica de los ríos regulados (SABATER $e t$ al., 1993), aunque se han comprobado descensos en la riqueza específica y eri la diversidad de las comunidades de inacroinvertebrados acuáticos. así como tina reducción en las áreas de distribución de la anguila, el salmón y la trucha (GARCÍA DE JALÓN et al., 1992). En el ámbito riojano, la información ecológica sobre ríos regulados es pidcticaincntc nula, aunque se dispone de algunos datos sobre el rógimen hidrológico (GARCÍA RUIZ \& MARTÍN RANZ, 1992).

La reciente construcción del embalse de Pajares, en la cabecera del río Iregua (La Rioja), representaba una 


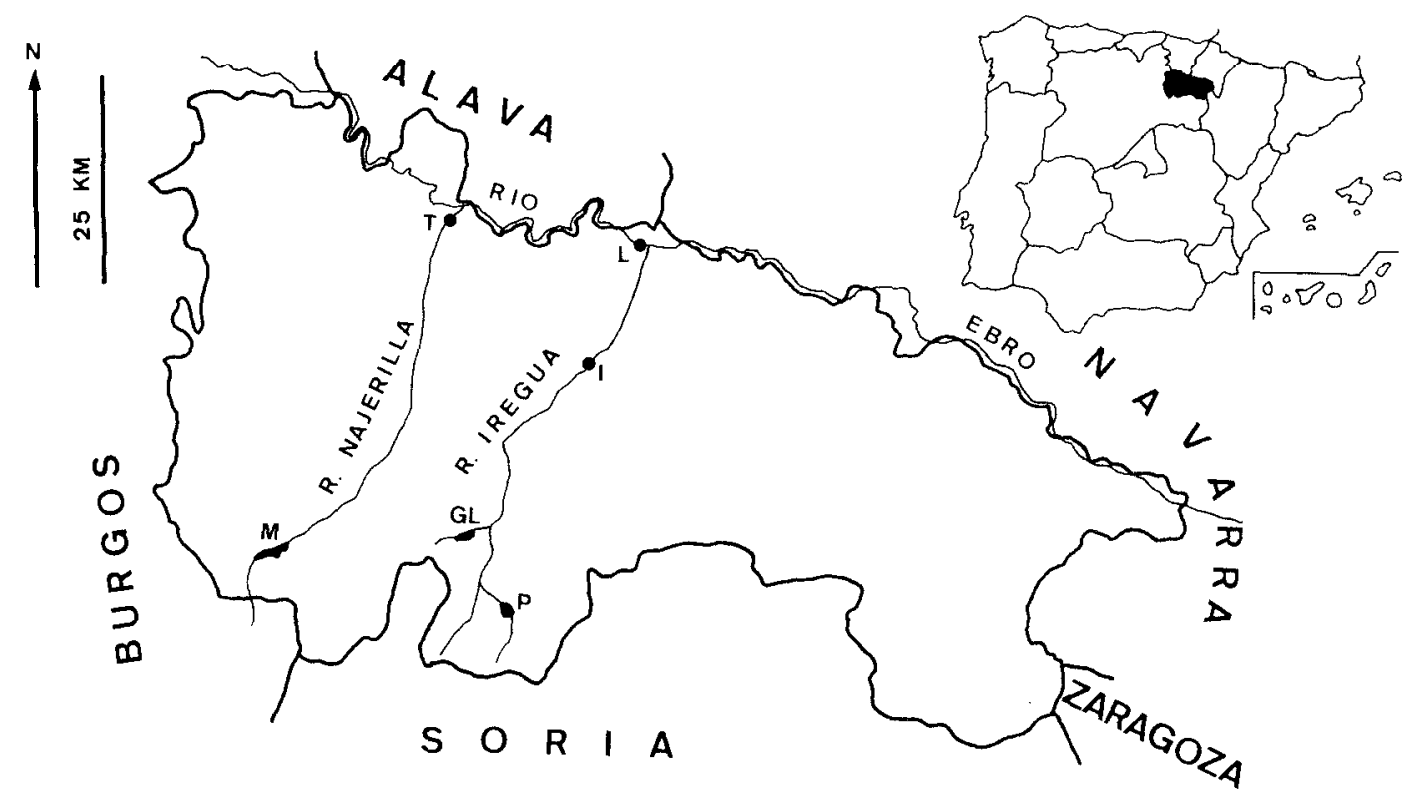

Fig. 1. Situación geográfica de los ríos lregua y Najerilla, las estaciones de análisis físico-químico (Islallana, I, y Torremontalbo, T) y los embalses de regulación (González Lacasa, GL: Pajares. P: Mansilla, M). L. Logroño.

Fig 1. Geographical situation of the rivers Iregua aiid Najerilla, the sampling stations (Islallana, I, and Torremontalbo. T) and the reservoirs (González Lacasa, GL: Pajares, P: Mansilla, M). L. Logroño.

posibilidad de experimentación sobre el iinpacto que genera una obra pública de estas características sobre un río ya previamente regulado a través de un embalse en un arroyo tributario. Sin embargo, no se ha realizado un seguimiento especial dc las características físico-químicas o biológicas del río Iregua durante el desarrollo de las obras. Nuestro trabajo pretende, a partir de los datos físico-químicos disponibles, analizar el impacto que ha tenido la construcción del embalse sobre las características del agua del río Iregua

\section{METODOLOGÍA}

\section{Área de estudio}

La cuenca del río Iregua ocupa $692 \mathrm{~km}^{2}$ de la parte central de la comunidad autónoma de La Rioja (Fig. 1). El curso principal del río, con una vaga orientación $\mathrm{S}-\mathrm{N}$, tiene una longitud de $62 \mathrm{~km}$, desde su nacimiento en la Sierra Cebollera, alrededor de los $2.000 \mathrm{~m}$ de altitud, hasta su desembocadura en las cercanías de Logroño, a $380 \mathrm{~m}$. La litología de la cuenca es variada (IGME, 1980). La cuenca alta cstá formada por materiales mesozoicos del Jurásico y Cretácico en facies Purbeck-Weald, en la que dominan las cuarzarenitas y las areniscas, con intercalaciones de calizas, margas y limolitas. La cuenca media está constituida por calizas del Jurásico inarino y dolomías, calizas, arcillas y yesos del Triásico. La parte baja está formada por conglomerados calizos del Terciario, excepto en el cauce del río y sus proximidades, donde se hallan materiales detríticos del Cuaternario.

En las cuencas alta y media predominan las actividades forestales, la ganadería extensiva y la agricultura de montaña. La presión poblacional en estas zonas es escasa. En contraste, en la cuenca baja se asientan los mayores núcleos de población, ligados a actividades de agricultura intensiva y extensiva, así como a una incipiente actividad industrial agroalimentaria.

El régimen hidrológico del río lregua presenta en cabecera un señalado carácter pluvionival, que se va atenuando a lo largo del curso (GARCÍA RUIZ \& MARTÍN RANZ, 1992). El Iregua se encuentra regulado por el embalse de González Lacasa, en Ortigosa de Cameros (Fig. 1), desde la década de los 50. Aunque proyectado algunas décadas antes, el embalse de Pajares no comenzó a construirse hasta el año hidrológico 1986-87, y las obras de construcción se prolongaron hasta 1994. En estos años se realizaron actividades de 
dcforcstación del vaso, reposición de viales, extracción de materiales, modificaciones en cauces de ríos, levantamiento de la presa, etc. Por último, a partir de 1995 se comenzaron a hacer las maniobras experimentales de embalse y desembalse.

\section{Datos físico-químicos utilizados}

El río lregua cuenta con varias estaciones de aforo (GARCÍA RUIZ \& MARTÍN RANZ, 1992) y actualmente con dos estaciones de análisis físico-químico sistemático (Islallana a $580 \mathrm{~m}$ y Albelda a $500 \mathrm{~m}$ ), controladas por la Comisaría de Aguas de la Confederación Hidrográfica del Ebro. La scric de datos más completa corresponde a Islallana, ya que Albelda sólo tiene datos disponibles a partir de 1993. Los análisis que se realizan en Islallana incluyen, con periodicidad mensual, las siguientes variables: caudal, temperatura del agua, $\mathrm{pH}$, conductividad, materiales en suspensión, oxígeno disuelto (cantidad y porccntajc de saturación), demanda química de oxígeno (DQO), demanda bioquímica de oxígeno (DBO5) y alcalinidad. En general, dos veces al año se realizan los siguientes análisis complementarios: calcio, magnesio, sodio. zinc, cobre, hierro, inangancso, arsénico. cadmio, cromo, plomo, amonio, cloruros, sulfatos, nitratos, nitritos, fosfatos, detergentes, fenoles, cianuros, fluoruros y coliforincs totales. El período utilizado ha sido 1981-1995, lo cual permite tener una visión suficientemente comprensiva de las características físico-químicas del río Iregua previamente a la construcción del embalse, a la vez que permite evaluar los cambios surgidos tras el comienzo de las obras.

En una primera revisión de los datos de Islallana, se apreciaban cambios en ciertas variables que sugerían un cfccto directo de las obras del embalse. Para comprobar esta relación causa-efecto, se incluyeron también en el estudio los datos analíticos de una estación del río Najerilla con características similares a Islallana, que pudiera servir corno estación control de cambios simultáneos en las dos cucricas provocados por causas ajenas al embalse. De las estaciones disponibles en el Najerilla, se eligió Torremontalbo, a $429 \mathrm{~m}$ de altitud (Fig. 1), por tener datos disponibles en el mismo período que lslallana y por cierta similaridad de características geográficas. Sus datos analíticos se obtuvieron de la misma forma que los de Islallana.

Dada la gran variabilidad temporal de algunos parámetros, se optó por obtener y representar también el suavizado de los datos. calculado mediante media móvil de 20 términos.

\section{RESULTADOS Y DISCUSIÓN}

La evolución de las variables seleccionadas en el período 1981-1995, tanto en su variabilidad natural como en el suavizado de los datos, se refleja en las Fig. 2 a 8 . El caudal (Fig. 2) es sensiblemente mayor en Torremontalbo que en 1slallana en todo el período estudiado, como consecuencia de la mayor pluviosidad que se registra en la cuenca del Najerilla (NÚÑEZ OLIVERA \& MARTÍNEZ ABAIGAR, 1991) y la regulación de ambos ríos por embalses, lo que probablemente atenúa los valores máximos y mínimos de caudal. En ambas localidades se observa un patrón estacional, con valores máximos en primavera temprana y con estiajes acusados, a pesar de la citada regulación. Este modelo de variación es similar al de otros ríos de la cuenca mediterránea (SABATER et al., 1993). Se aprecia un cierto paralelismo entre las dos localidades a lo largo del período, como consecuencia de la alternancia de períodos plurianuales secos y húmedos (NÚÑEZ OLIVERA \& MARTÍNEZ ABAIGAR, 1991). En ambas localidades, las variaciones estacionales se atenúan a partir de 1993.

La evolución de la temperatura del agua (Fig. 3), con temperaturas generalmente más frías en 1slallana que cn Torremontalbo, es consecuencia, fundamentalmente, de la mayor altitud de Islallana, dado que existe una estrecha correlación negativa entre la temperatura del aire y la altitud (NÚÑEZ OLIVERA \& MARTÍNEZ ABAIGAR, 1991) y una correlación positiva entre la temperatura del agua y la del aire (WALLING \& WEBB, 1992). Otra causa adicional es que el clima del valle del Iregua es más frío, para altitudes similares. que el clima del valle del Najerilla (NÚÑEZ OLIVERA \& MARTÍNEZ ABAIGAR, 1991). Al igual que en el caso del caudal, la temperatura tiene cambios estacionales evidentes. A partir de 1992 la temperatura del agua de 1slallana asciende hasta casi equilibrarse con la de Torremontalbo, sin que ésta registre cambios apreciables. El drástico e inexplicado ascenso de la temperatura del aire en lslallana (datos no representados) es el responsable de dicha alteración.

Salvo algunos picos puntuales, las variaciones en el $\mathrm{pH}$ (Fig. 4) son ligeras, tanto en Islallana como en Torremontalbo. En ambos ríos los valores de $\mathrm{pH}$ tienden a una cierta alcalinidad, de acuerdo con la litología predominantemente caliza de las cuencas medias y bajas del Iregua y el Najerilla, y también a causa de la situación en cuenca baja de ambas estaciones de muestreo. Los cambios en la conductividad (Fig. 5) son asimismo poco significativos. Los valores del Najerilla son más altos que los del Iregua, probablemente debido a la mayor superficie de cuenca y al mayor caudal, que ocasionan una mayor carga iónica. Las variaciones estacionales apuntan 

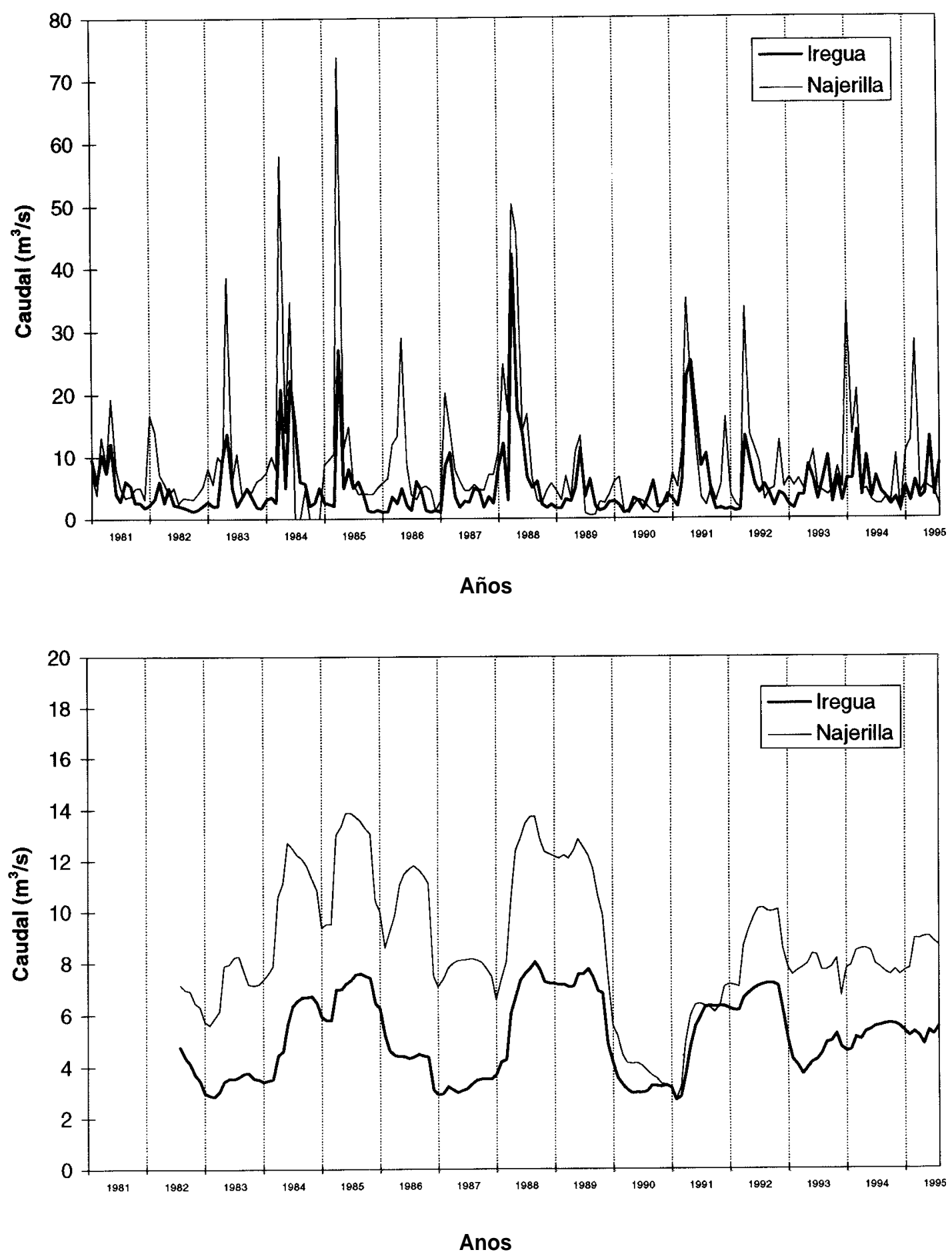

Fig. 2. Evolución temporal del caudal en los ríos lregua (estación de Islallana) y Najerilla (estación de Torremontalbo) durante el periodo 1981-1995. Se presenta la cvolución de los datos brutos (arriba) y de los datos suavizados mediante media móvil (ahajo).

Fig. 2 Temporal changes of the water flow of the rivers Iregua (Islallana) and Najerilla (Torremontalbo) during the period 1981-1995. Both roiigh data (above) and srnoothed data by mobile average (below) are shown. 


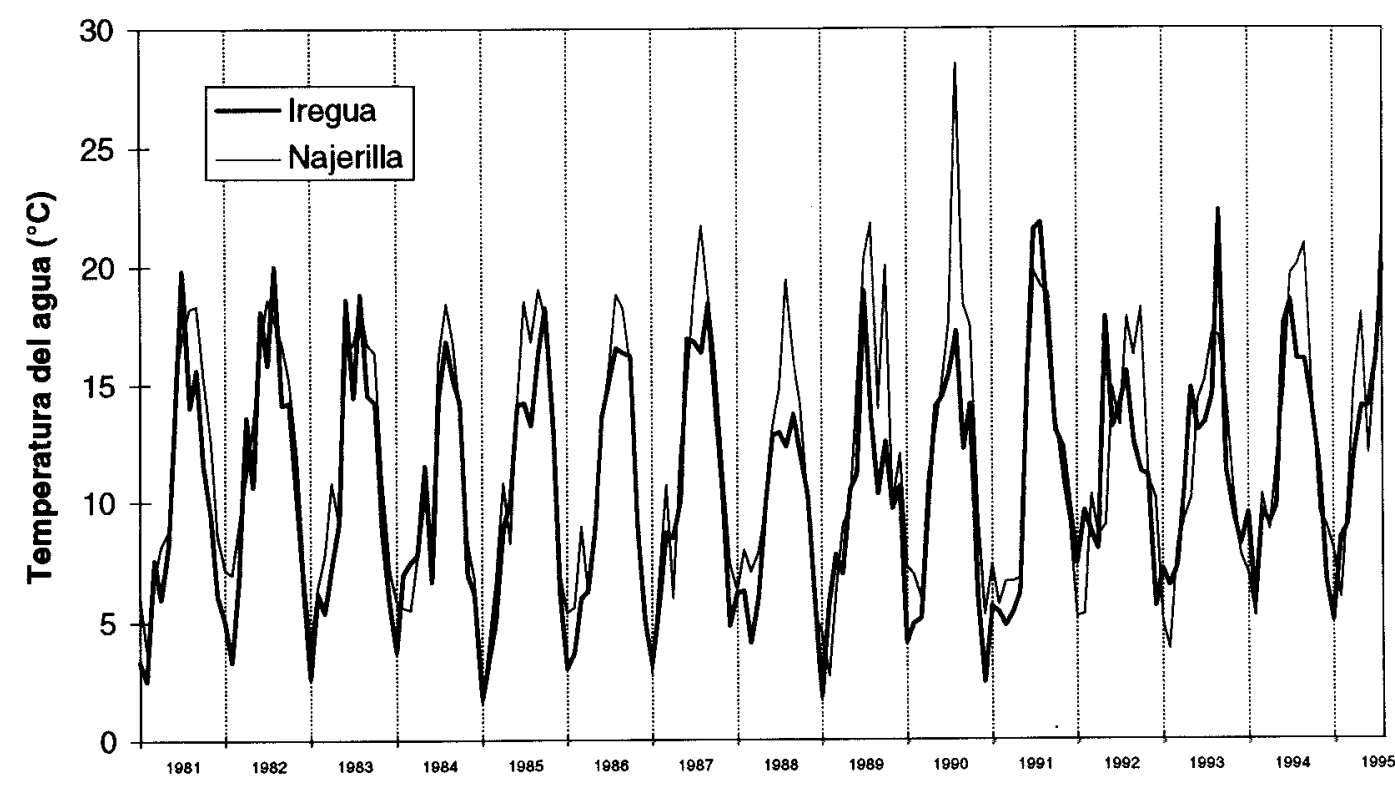

Años

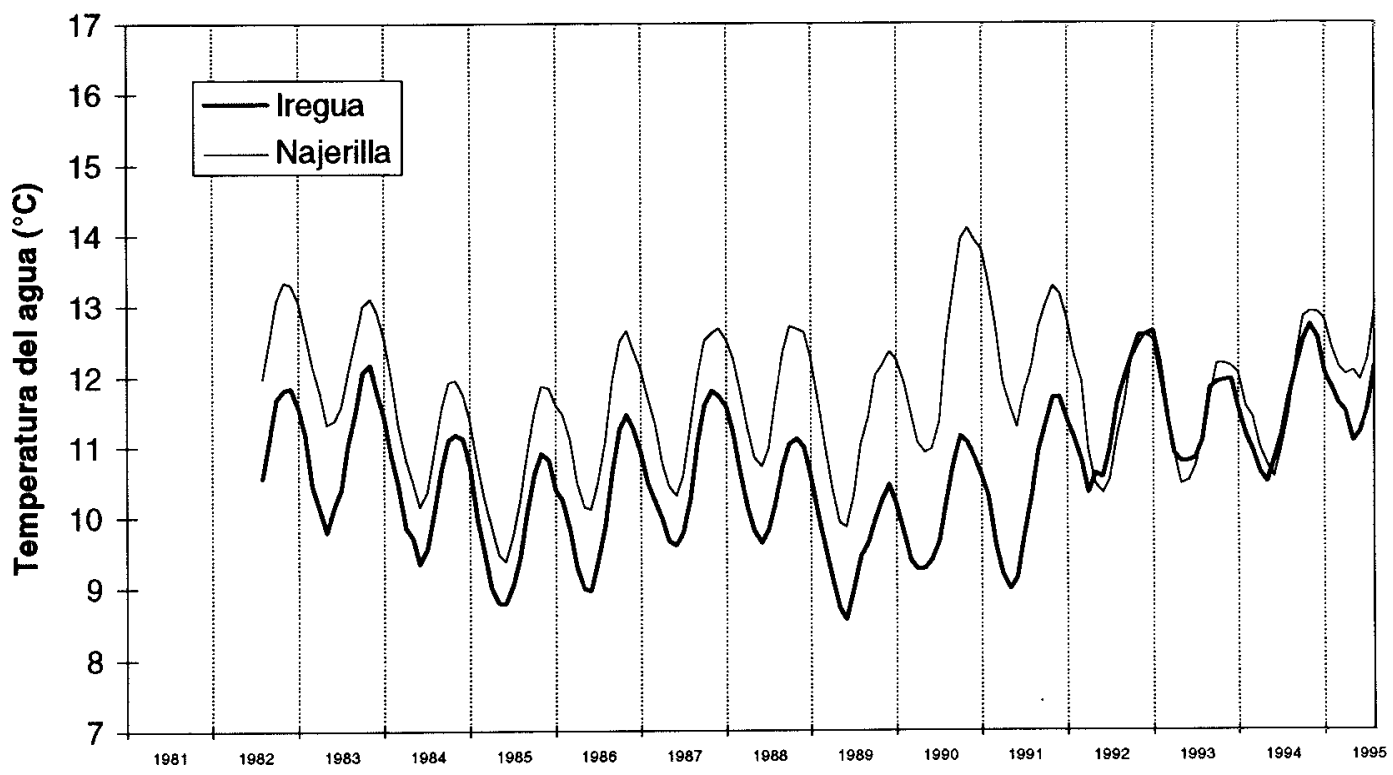

Años

Fig. 3. Evolución temporal de la temperatura del agua en los ríos lregua (eslación de Islallana) y Najerilla (estación de Torremontalbo) durante el periodo 1981-1995. Se presenta la evolución de los datos brutos (arriba) y de los datos suavizados mediante inedia móvil (abajo)

Fig 3 Temporal changes of the water temperature of the rivers Iregua (Islallana) and Najerilla (Torremontalbo) during the period 1981-1995 Both rough data (above) and smoothed data by mobile average (below) are shown. 
generalmente a aumentos en la conductividad en el estiaje, por la concentración de solutos, aunque algunos picos aislados pueden explicarse por la incidencia de precipitaciones copiosas, que provoquen lavado de suelos (MARTÍNEZ ABAIGAR et al., 1993).

Las variaciories en los materiales en suspensión son sumamente interesantes (Fig. 6). El Najerilla lleva en torno a diez veces la cantidad de materiales que el Iregua, de acuerdo con su mayor caudal (SABATER et al., 1993), que conlleva una mayor fuerza erosiva y de arrastre, y la inayor superfice de cuenca. Dc todas formas, hay que tener en cuenta que, en los ríos regulados, los embalses funcionan como balsas de decantación y. por lo tanto. la cantidad de sólidos en suspensión disminuye drásticamente a la salida del embalse (GARCÍA DE JALÓN et al., 1992). En ambos ríos los cambios en materiales suspendidos se correlacionan estrechamente con las variaciones de caudal en el período 1981-1990. A partir de esta fecha, los valores en el Najerilla se mantienen relativamente homogéneos y bajos, a pesar de que el caudal registra ciertas variaciones (aunque no tan acusadas como en dicho período). Sin embargo, en el Iregua se observa un acusado aicenso de materia en suspensión a partir de 1991. sin un ascenso claro y simultárieo de caudal. Es probable que este ascenso registrado en los materiales en suspensión en la estación de Islallana se deba a los movimientos de tierra efectuados en las obras del embalse de Pajares. De hecho, el aporte de sedimentos a la corriente fluvial es uno de los impactos habituales en la construcción de embalses (DIRECCIÓN GENERAL DE MEDIO AMBIENTE, 1989). La notable disiancia del embalse a Islallana habría ocasionado una atenuación del impacto a lo largo del curso del río. pero a pesar de ello todavía es apreciable. La acumulación de materia en suspensión y el consiguiente aurnento de turbidez provocan notables alteraciones en el medio acuático: reducción de la intensidad luminosa y variación del espectro (WESTLAKE, 1966; JEFFREY, 1981), aurnento de los daños por abrasión en las comunidades sumergidas (WALLING \& WEBB, 1992; MARTÍNEZ ABAIGAR et al., 1993), etc. Además, el aumento de turbidez genera impactos visuales poco deseables.

El oxígeno disuelto (Fig. 7) es generalmente mayor en el Najerilla que cn el Iregua, probableinente por su inayor caudal, que favorece la turbulencia y, por tanto, la aireación. Además. la mayor presión atmosférica de Torremontalbo respecto de Islallana aumenta la concentración de equilibrio del oxígeno (WALLING \& WEBB, 1992). Sin embargo, en los últimos años los valores de ambos ríos tienden a solaparse, quizá por las variaciones poco explicables en la temperatura (Fig. 3). De todas formas, las aguas del Iregua y el Najerilla en las estaciones de muestreo son aguas oxigenadas. sin problemas de anoxia ni de metabolismo heterótrofo. En ambos ríos se registran patrones estacionales de variación, eri relación con el caudal y la temperatura. Los valores mínimos se dan en los meses de verano, por el escaso caudal (que limita la renovación de gases por turbulencia) y la temperatura alta (la solubilidad del oxígeno disminuye con el aurnento de temperatura: MARGALEF, 1983). Por el contrario, los máximos anuales suelen alcanzarse en primavera o invierno, con caudales altos y temperaturas medias. La DBOs se mantiene más alta en el Najerilla que en el lregua hasta 1991 (Fig. 8), aunque, salvo excepciones relacionadas a priori con el caudal, los valores registrados no suponen problemas graves de anoxia en las aguas (MARGALEF, 1983; MARTÍNEZ ABAIGAR et al., 1993). A partir de 1991 la variación se invierte y la DBOs del lregua alcanza o supera a la del Najcrilla, que también aumenta ligeramente. El origen de la inversión es probablemente doble; por una parte, el acusado aumento de materiales en suspensión en el Iregua podría haberse debido en cierta parte a material orgánico Por otro lado, las lluvias pueden favorecer la resuspensión de lodos orgánicos (MARTíNEZ ABAIGAR et al. 1993) y consecuentemente pueden hace que la DBOs se incremente en ambos ríos. La precipitación registrada en 1992, un 20\% superior a la normal (GOBIERNO DE LA RIOJA, 1993), podría apoyar este segundo punto; sin embargo, la precipitación de 1993 está por debajo de la media normal (GOBIERNO DE LA RIOJA, 1995).

El resto de las variables disponibles no han sido representadas, bien porque resultaban redundarites o bien por la escasa representatividad de sus datos. Así, las variaciones en la alcalinidad y los cationes y aniones principales responden básicamente a las variaciones en la conductividad. Los metales pesados que poseen series de datos representativas (hierro, manganeso. zinc, cobre) tampoco ofrecen información significativa, al igual que los nitratos, riitritos, fosfatos. detergentes, fluoruros y coliformes totales.

En conclusión. la construcción del embalse de Pajares originó, a partir de 1991, un acusado ascenso de materia en suspensión en el río Iregua. tomando como base los análisis de la propia Conlederación Hidrográfica. Este impacto se habría visto atenuado a lo largo del curso del río hasta Islallana, la estación de muestreo. Los electos del aurnento de materia en suspensión sobre las comunidades acuáticas son difícilmente valorables, puesto que se carece de inventarios previos (salvo en el caso de los briófitos: MARTÍNEZ ABAIGAR \& NÜÑEZ OLIVERA, 1991) y tampoco se han realizado estudios posteriores sobre la posible recuperación de dichas co 

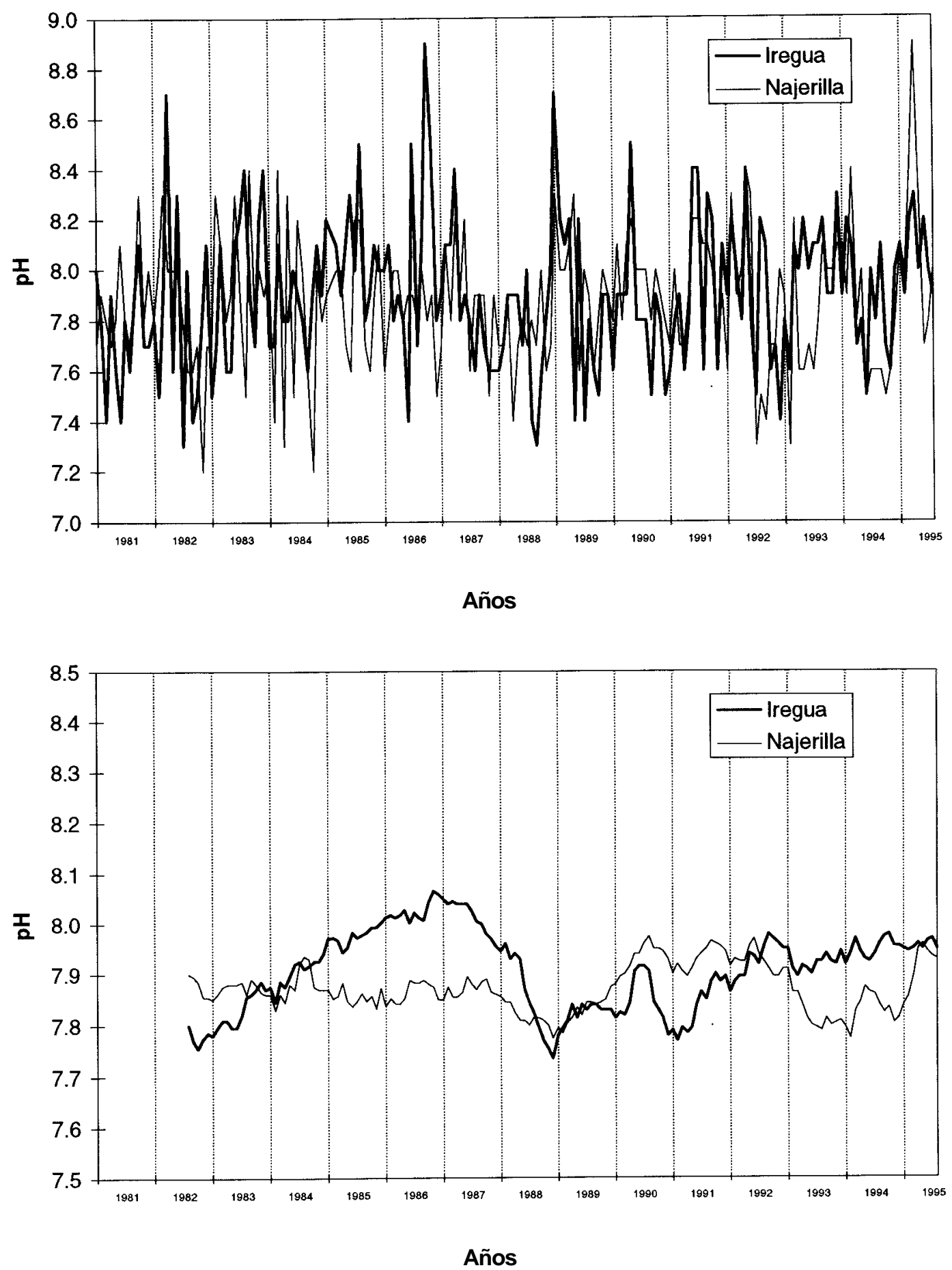

Fig. 4. Evolución temporal del pHen los ríos Iregua (estación de Islallana) y Najerilla (estación de Torremontalbo) durantc el periodo 1981-1995. Se presenta la evolución de los datos brutos (arriba) y de los datos suavizados mediante media móvil (abajo).

Fig. 4. Temporal changes of the water $\mathrm{pH}$ of the rivers Iregua (Islallana) and Najerilla (Torremontalbo) during the period 1981-1995. Both rough data (above) and smoothed data by mobile average (below) are shown. 


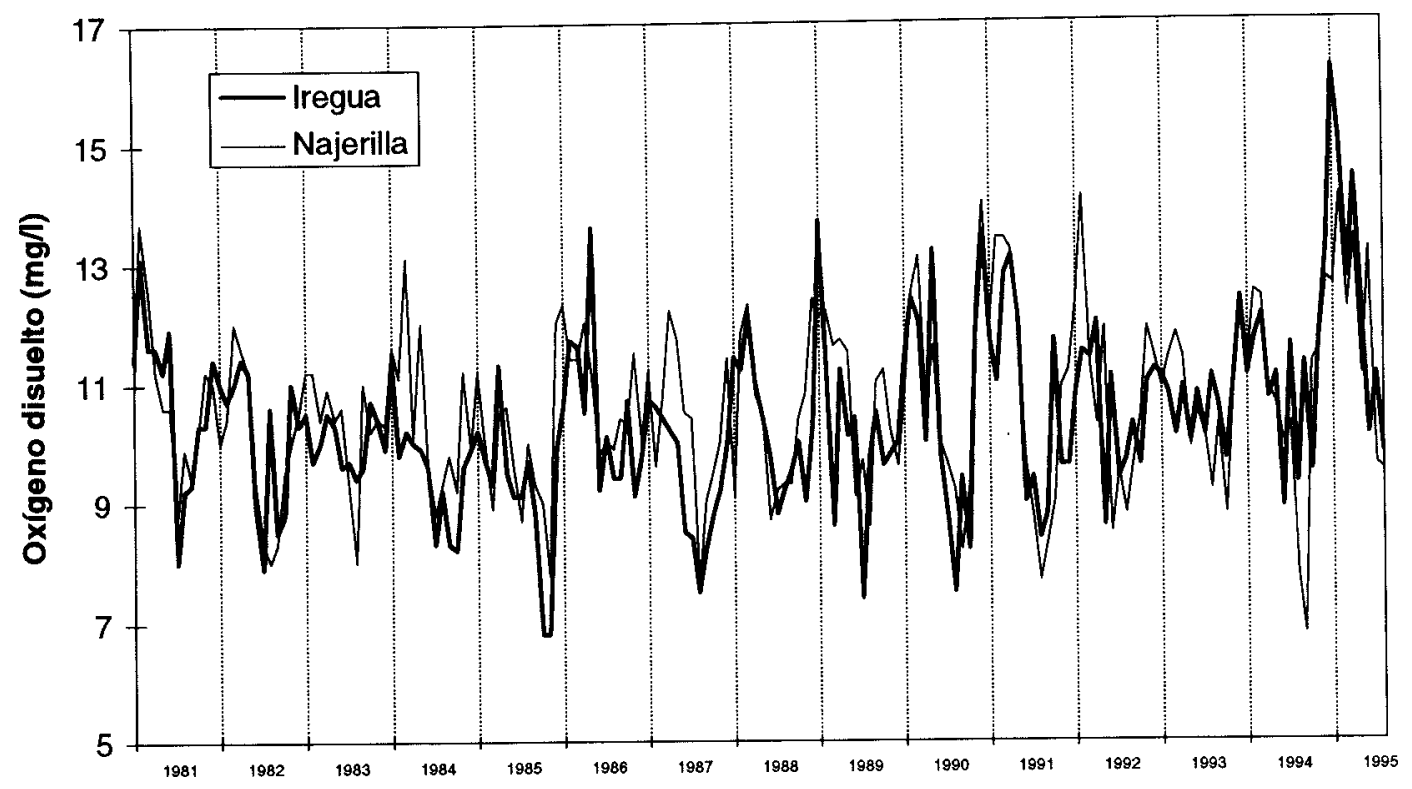

Años

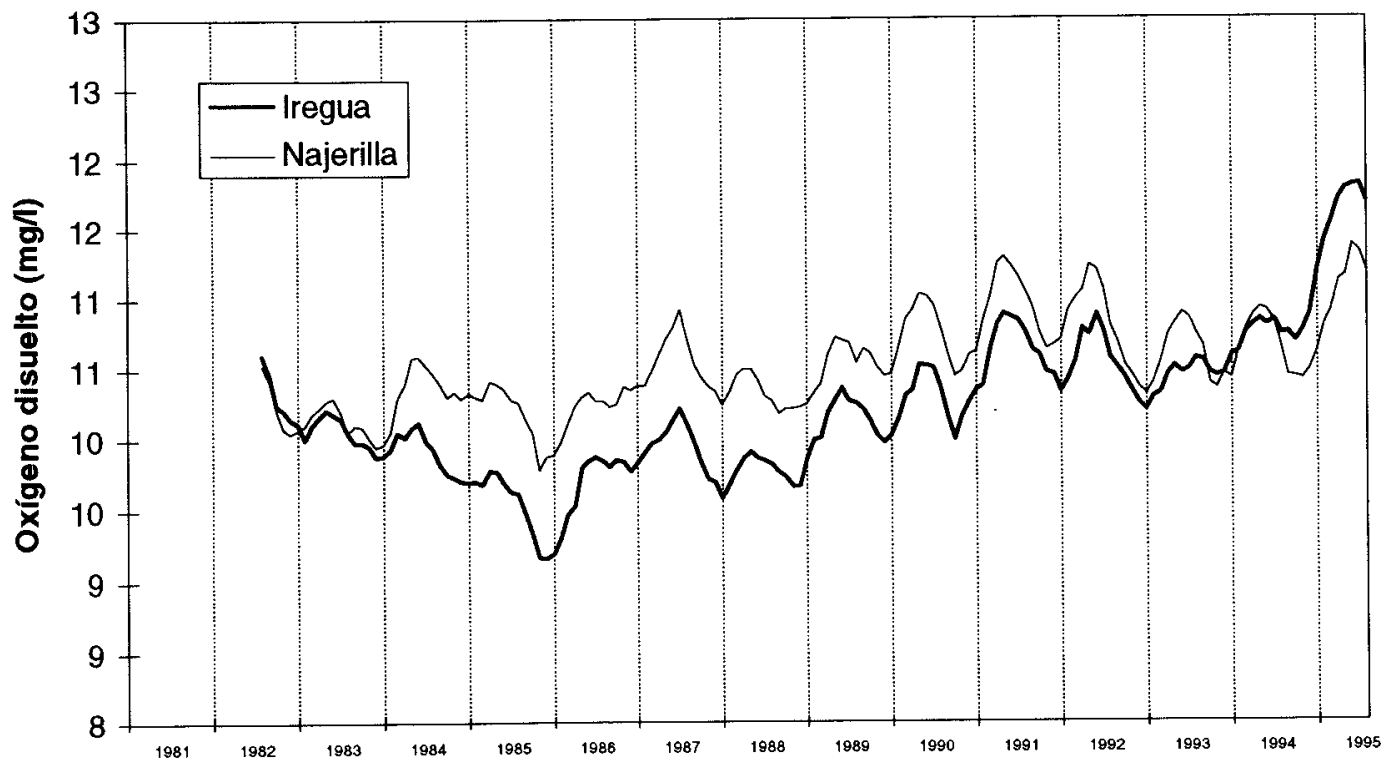

Años

Fig. 5. Evolución temporal de la conductividad en los ríos Iregua (estación de Islallana) y Najerilla (estación de Torremontalbo) durante el periodo 1981-1995. S r presenta la evolución de los datos brutos (arriba) y de los datos suavizados inediante media móvil (abajo).

Fig. 5 . Temporal changes of the water conductivity of the rivers Iregua (Islallana) and Najerilla (Torremontalbo) during ihe period 1981-1995. Both rough data (abovc) and smoothed data by mobile average (below) are shown. 
Materia en suspensión (mgll) IREGUA

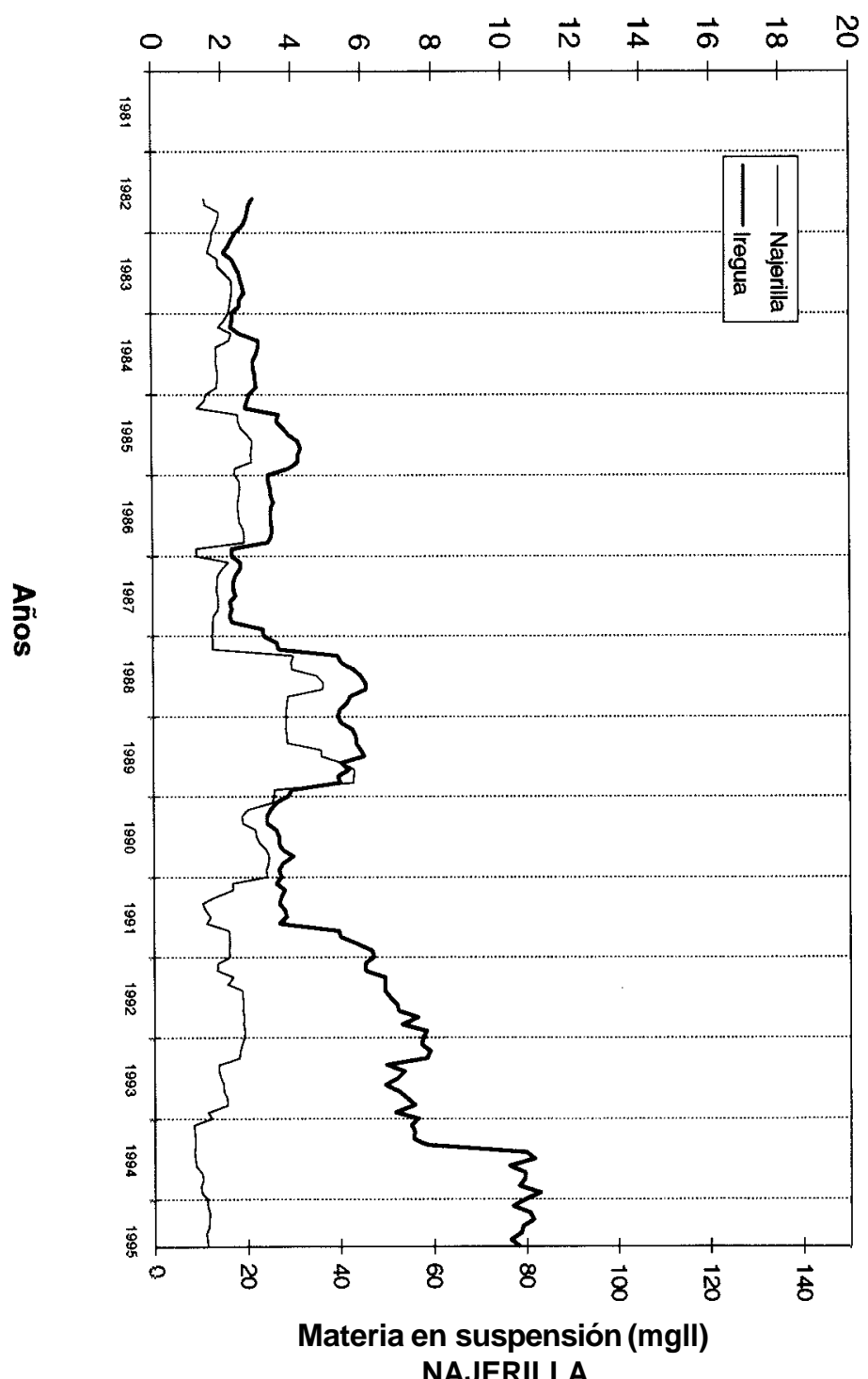

Materia en suspensión (mgll)

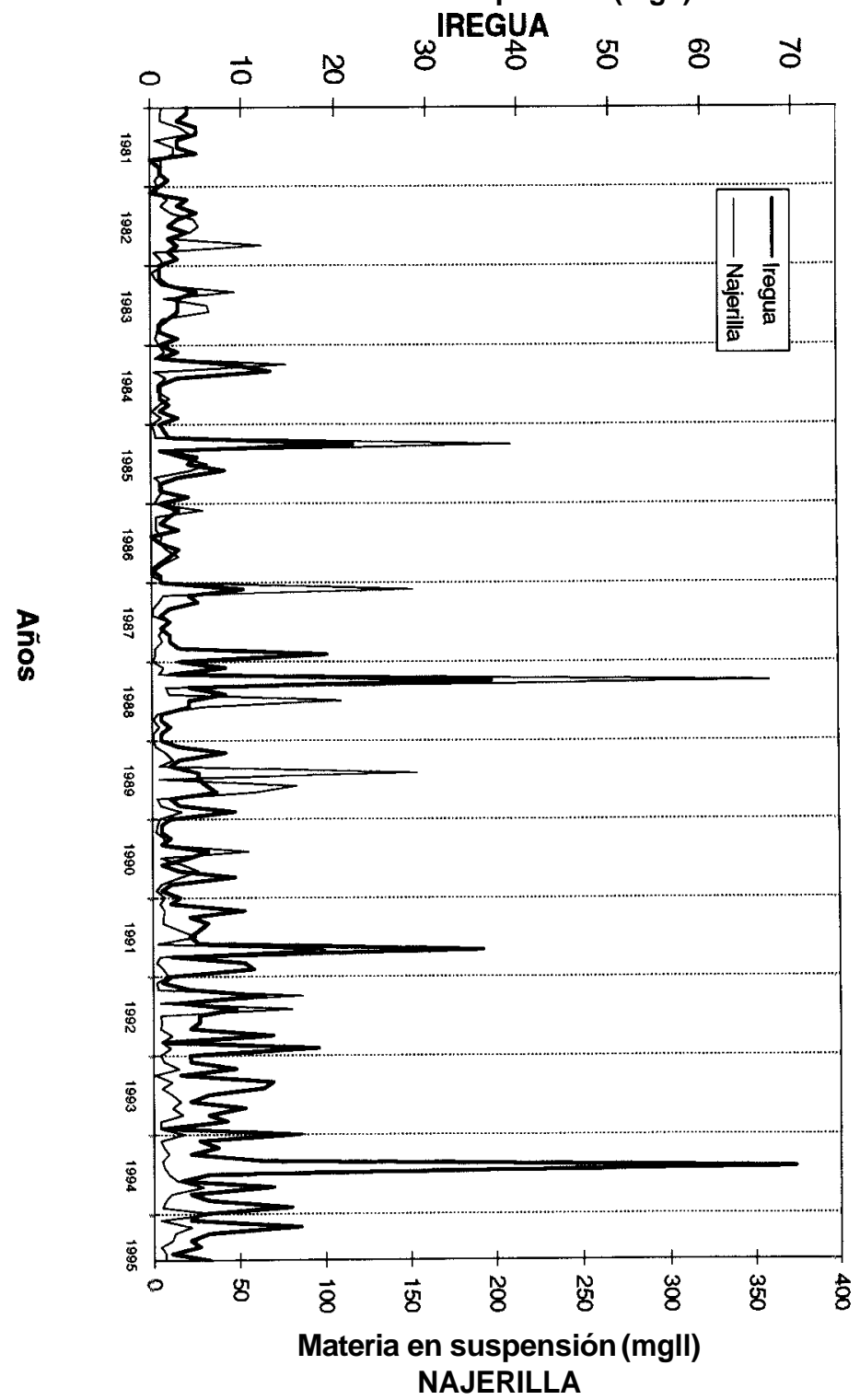


10

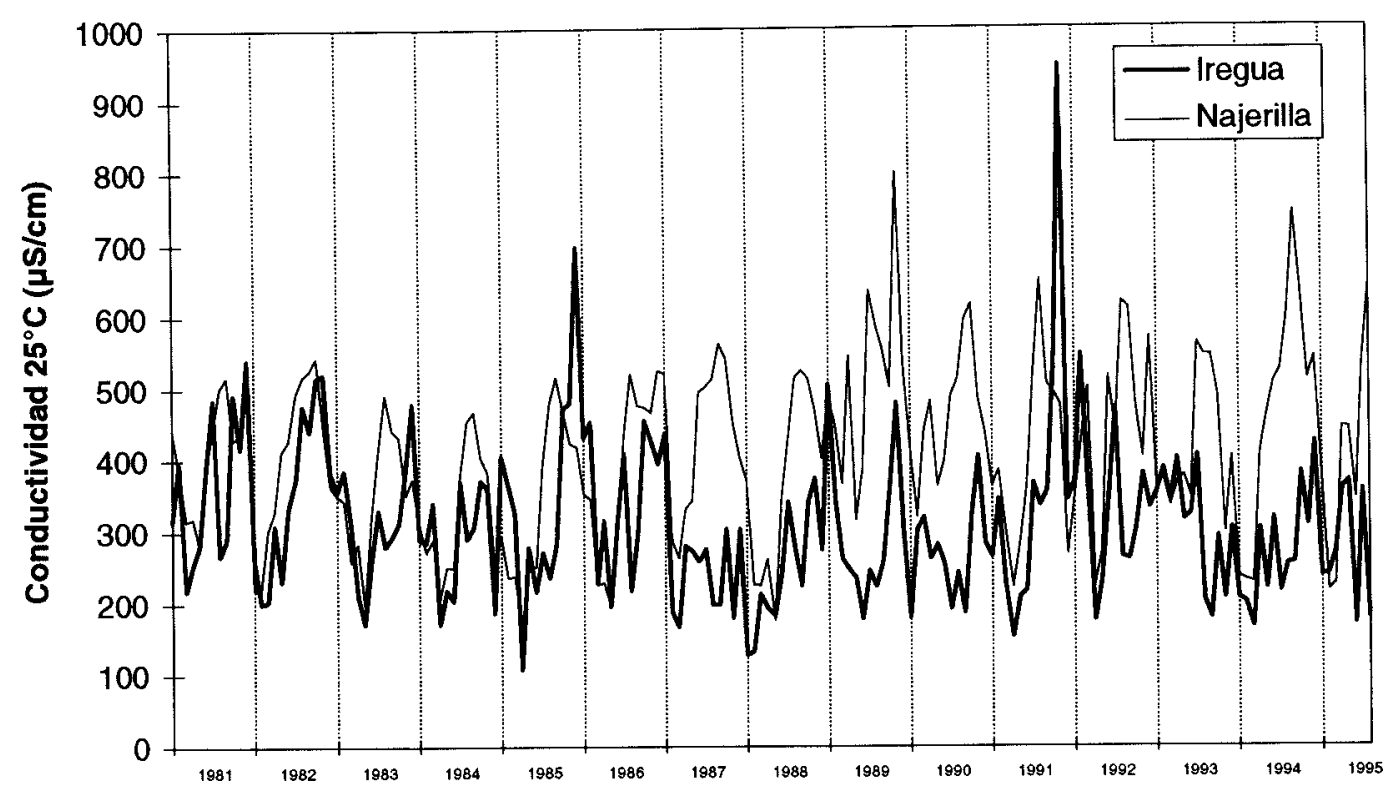

Años

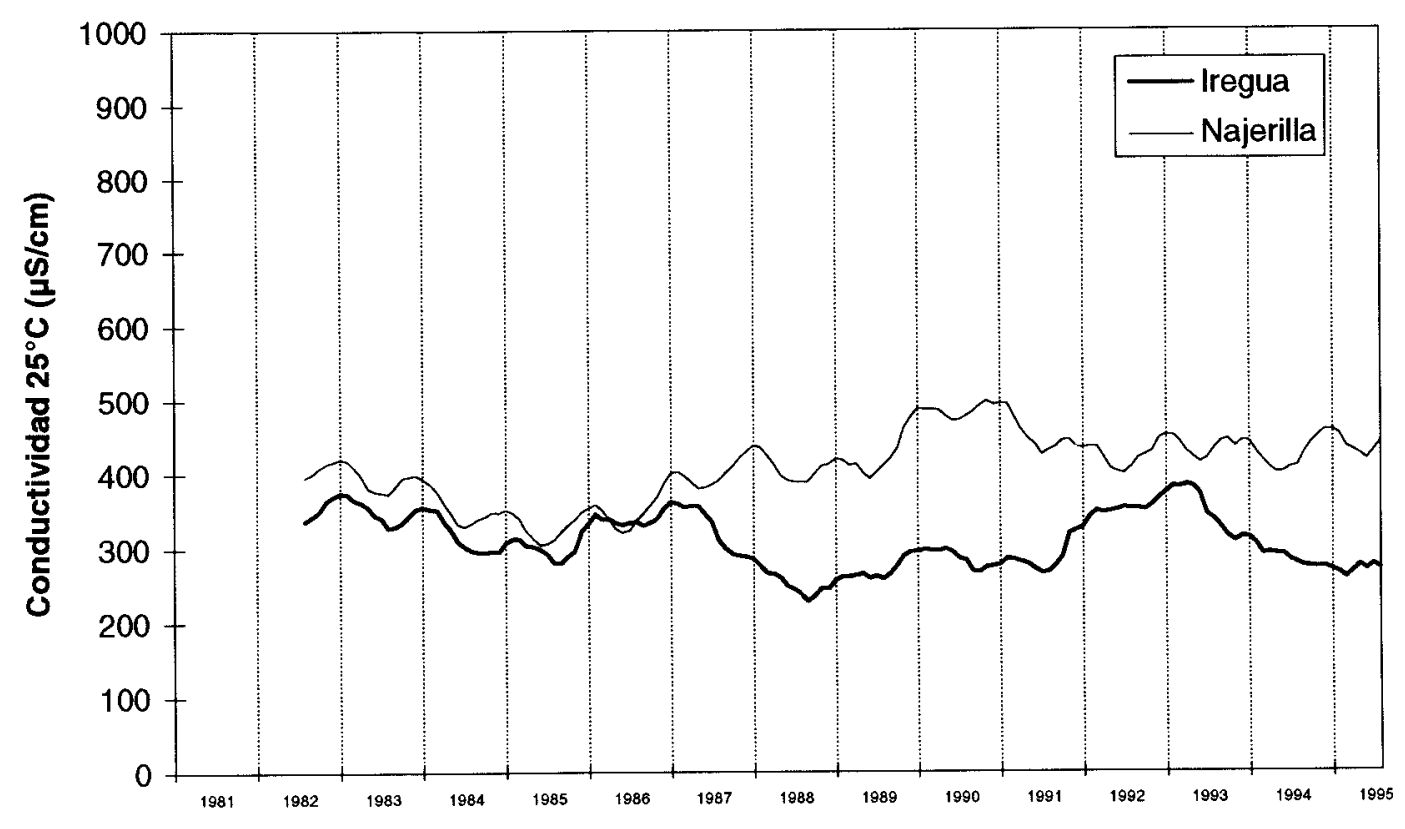

Años

Fig. 7. Evolución temporal de la concentración absoluta de oxígeno disuelto en los ríos Iregua (estación de Islallana) y Najerilla (estación de Torremontalbo) durante el periodo 1981-1995. Se presenta la evolución de los datos brutos (arriba) y de los datos suavizados mediante rnedia móvil (abajo).

Fig. 7. Temporal changes of the dissolved oxygen of the rivers Iregiia (Islallana) and Najerilla (Torremontalbo) during the period 1981-1995. Botli rough data (above) and smoothed data by mobile average (below) are shown. 

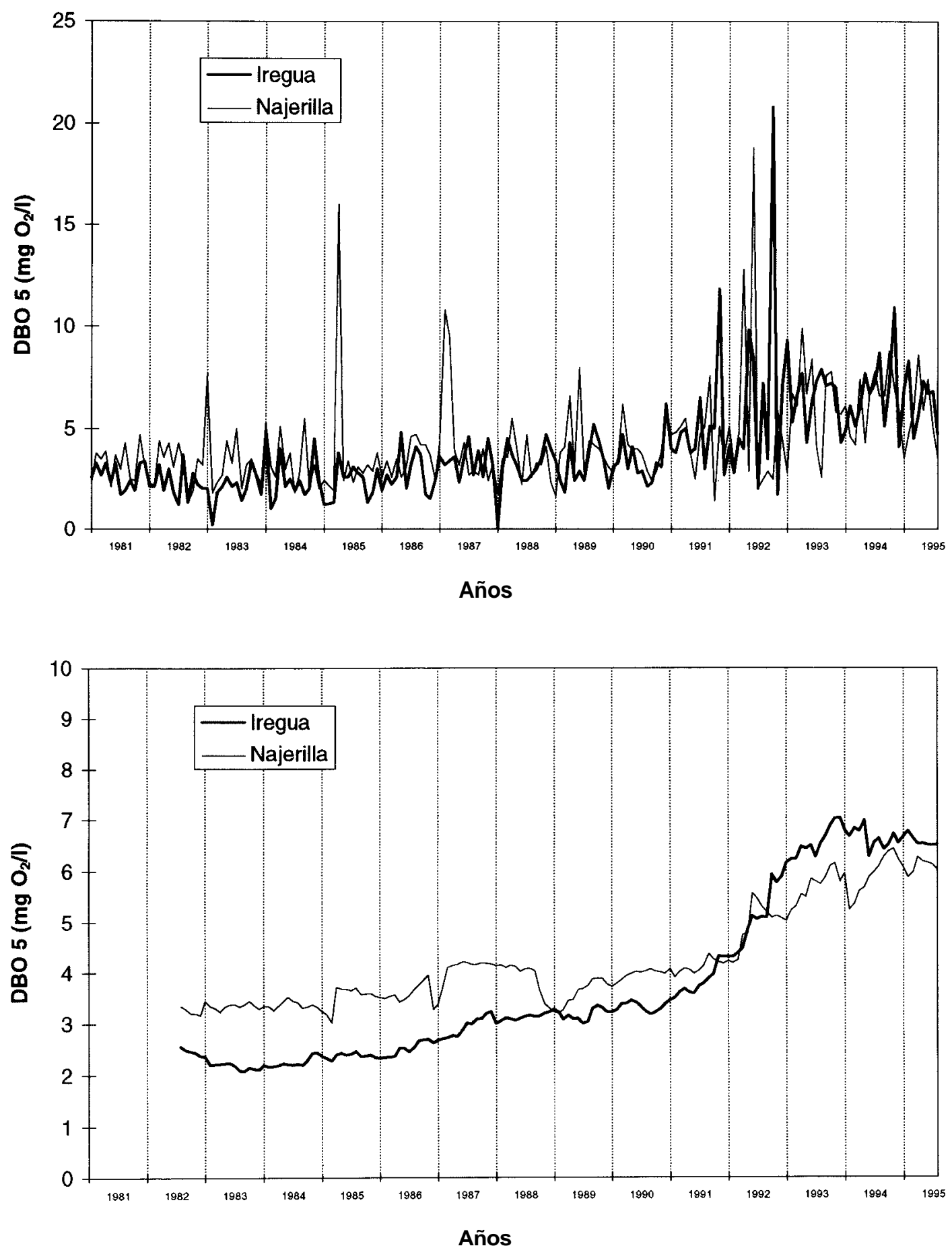

Fig. 8. Evolución temporal de la demanda bioquímica de oxígeno (DBO5) en los ríos Iregua (estación de Islallana) y Najerilla (estación de Torremontalbo) durante el periodo 1981-1995. Se presenta la evolución de los datos brutos (arriba) y de los datos suavizados mediante media m- vil (abajo).

Fig S. Temporal changes of the biochemical oxygeii demand (BOD5) of the rivers Iregua (Islallana) and Najerilla (Torremontalbo) during the period 1981-1995. Both rough data (above) and smoothed data by mobile average (below) are shown. 
munidades. Tomando como control lo estación de Torremontalbo, en el río Najerilla, se aprecian también cambios en otras variables (temperatura, oxígeno. DBO5) en la estación de Islallana a partir del período 1991-1992. Es posible que algunos de estos cambios se relacionen con las variaciones en los materiales en suspensión.

\section{AGRADECIMIENTOS}

A la Confederación Hidrográfica del Ebro (Sección Comisaría de Aguas) por suministrarnos los datos analíticos inéditos. Este trabajo se enmarca dentro del Proyecto CICYT AMB95-0468, del Plan Nacional de I+D.

\section{BIBLIOGRAFÍA}

DIRECCIÓN GENERAL DE MEDIO AMBIENTE, 1989. Guías metodológicas para la elaboración de estudios de impacto ambiental 2. Grandes presas. Monografías de la Dirección General de Medio Ambiente, Ministerio de Obras Públicas y Urbanismo. Madrid.

GARCÍA DE JALÓN, D., GONZÁLEZ DEL TÁNAGO, M. \& C. CASADO. 1992. Ecology of regulated streams in Spain: an overview. Limnetica 8: 161-166.

GARCÍA RUIZ, J.M. \& M.C. MARTÍN RANZ. 1992. El régimen de los ríos de La Rioja. Instituto de Estudios Riojanos. Logroño.

GOBIERNO DE LA RIOJA, 1993. Estadística agraria regional 1992. Conscjería de Agricultura y Alimentación. Logroño.

GOBIERNO DE LA RIOJA, 1995. Estadística agraria regional 1993. Consejería de Agricultura y Alimentación. Logroño.

IGME (Instituto Geológico y Minero de España), 1980. Mapa Geológico de España E. I:200.000. Síntesis de la Cartografia existente. Hoja no 21. Logroño. Servicio de Publicaciones, Ministerio de Industria y Energía. Madrid.

JEFFREY, S.W., 1981. Responses to light in aquatic plants. In: Physiological Plant ECOIORY 1: Responses ro the phrsical
Environment. Encyclopedia of Plant Physiology New Series (O.L. LANGE, P.S. NOBEL, C.B. OSMOND \& H. ZIEGLER, eds.) 249-276. Springer-Verlag. Berlin.

MARGALEF, R., PLANAS, D., ARMENGOL, J., VIDAL, A., PRAT, N., GUISET, A., TOJA, J. \& M. ESTRADA. 1976. Limnología de los embalses españoles. Dirección Gcneral de Obras Hidráulicas, Ministerio de Obras Públicas, Publ. nº 23. Madrid.

MARGALEF, R. 1983. Limnología. Oincga. Barcelona.

MARTÍNEZ ABAIGAR, J. \& E. NÚÑEZ OLIVERA. 1991. Briófitos acuáticos del río Iregua (La Rioja). Estudio florístico ecológico y ecofisiológico. Respuestas a la contaminación orgánica. Instituto de Estudios Riojanos. Gobierno de La Rioja. Logroño.

MARTÍNEZ ABAIGAR, J., NÚÑEZ OLIVERA. E. \& M. SÁNCHEZ-DÍAZ. 1993. Effects of organic pollution on transplanted aquatic bryophytes. Journal of Bryology 17: 553566.

NÚÑEZ OLIVERA, E. \& J. MARTÍNEZ ABAIGAR. 1991. El clima de La Rioja. Análisis de precipitaciones y temperaturas. Gobierno de La Rioja, Consejería de Agricultura y Alimentación. Logroño.

RIERA, J.L., JAUME, D., DE MANUEL, J.. MORGUI, J.A. \& J. ARMENGOL. 1992. Patterns of variation in the limnology of Spanish reservoirs: a regional study Limnetica 8: 111-123.

SAHATER, S.. SABATER. F. \& J. ARMENGOL. 1993. Ecología de los ríos mediterráncos. Investigación y Ciencia 203: 72-79.

WALLING. D.E. \& B.W. WEBB. 1992. Water quality I. Physical characteristics. In: The Rivers Handbook, Vol. I (P. CALOW, \& G.E. PETTS, eds.) 48-72. Blackwell Scicntific Publications. London.

WESTLAKE, D.F. 1966. The light climate for plants in rivers. In: LiMht as an ecological factor (R. BATNBRIDGE, G.C. EVANS \& O. RACKHAM, eds.) 99-1 19. Blackwell Scientific Publications. London. 